\title{
Reduced left atrial early strain rate following acute sleep deprivation: chance finding or chance to find out more on the conundrum of sleep and cardiovascular disease?
}

\author{
Micha T. Maeder
}

Received: 15 October 2012 /Revised: 23 October 2012 / Accepted: 2 November 2012 /Published online: 13 November 2012

(C) Springer-Verlag Berlin Heidelberg 2012

There is now a large and robust body of evidence showing that the presence of severe obstructive sleep apnea (OSA) is associated with an increased risk of hypertension, atrial fibrillation, heart failure, and death [1]. In addition, epidemiological studies have also revealed associations between self-reported short sleep duration in the absence of known OSA and several cardiovascular risk factors including hypertension [2] and diabetes [3]. In this context, a number of small mechanistic studies using acute sleep deprivation models have been undertaken in healthy humans to test the hypothesis that sleep deprivation per se, i.e., in the absence of OSA, may adversely affect the cardiovascular system. In these studies, acute sleep deprivation has been shown to increase heart rate [4]; to adversely affect heart rate variability [4-6]; to increase atrial electromechanical delay [7], P wave dispersion [8], and QT dispersion [9]; to increase plasma norepinephrine [5]; and to impair vascular function $[4,5]$. To date, there are very little data on the impact of sleep deprivation on cardiac function as assessed by echocardiography, however.

In their current paper in Sleep and Breathing, Acar et al. [10] present the results of an innovative study investigating the effects of acute sleep deprivation on left heart function, in particular left atrial function. The authors performed comprehensive transthoracic echocardiography in a cohort of 27 normal subjects both after a night of regular sleep and after a night of sleep deprivation. Sleep deprivation was induced by night work (one night with partial sleep deprivation), and the intervention (normal sleep versus sleep deprivation) was not randomized. The authors assessed left atrial function not only by standard volumetric parameters

\section{T. Maeder $(\square)$}

Cardiology Division, Kantonsspital St. Gallen,

Rorschacherstrasse 95,

9007 St. Gallen, Switzerland

e-mail: micha.maeder@kssg.ch but also measurement of tissue Doppler-derived left atrial strain and strain rate. Strain reflects intrinsic myocardial deformation, and strain rate represents its change over time $[11,12]$. Both measurements have the advantage to be relatively load-independent. Although measurement of strain and strain rate on the atrial level is not currently in routine clinical use, a number of studies have demonstrated their applicability in different populations $[11,12]$. After a night of sleep deprivation (sleeping time $2.3 \mathrm{~h}$ versus $7.8 \mathrm{~h}$ during regular sleep), no significant effects on blood pressure, heart rate, left ventricular dimensions, left atrial dimensions, and volumetric measurements of left atrial function were found in the present study. However, the authors observed a significant reduction in left atrial early diastolic strain rate (a measure of left atrial conduit function) after sleep deprivation, and there was a moderate correlation between lower left atrial early diastolic strain rate and shorter sleep duration $\left(r^{2}=0.31\right)$. In contrast, there was no significant change in left atrial systolic strain, systolic strain rate, and late diastolic strain rate. There was also a small but significant increase in deceleration time and isovolemic relaxation time (measures of active left ventricular relaxation) after sleep deprivation. These are novel and interesting results, but we have to ask the following questions to properly put the findings into perspective: Are the findings real, or do they represent a chance finding? Are they biologically plausible? And finally, what are the clinical implications?

First, are the findings real, or do they represent a chance finding? The study is limited by the small sample size and the non-randomized intervention. However, the findings are rather convincing. The observed difference in left atrial early strain rate was small but statistically highly significant, whereas apart from deceleration time and isovolemic relaxation time, there was no difference in any of the other parameters. Importantly, as discussed below, the increase in deceleration time and isovolemic relaxation time following sleep deprivation also fit 
well into the pathophysiological concept of the study. As obvious from the individual data plots in Fig. 2 in the manuscript, all but three subjects had a lower left atrial early diastolic strain rate after sleep deprivation than after a night with regular sleep indicating that a chance finding is unlikely. The authors are to be commended for their efforts to obtain a homogeneous study population without conditions potentially confounding their measurements. All echocardiograms were obtained by the same physician, and intraobserver variability was acceptable. Although this is not exactly described in the manuscript, I assume that the physician analyzing the echocardiogram was blinded to whether or not a subject had had normal sleep or had sleep debt. Thus, the findings appear to be real. Still, the findings will have to be reproduced.

The next question is whether or not the findings are biologically plausible. In contrast to the highly significant reduction in left atrial early diastolic strain rate after sleep deprivation, there were no significant changes in left atrial systolic strain, systolic strain rate, and late diastolic strain rate. This fits very well to the isolated, small but significant increase in left ventricular relaxation and isovolemic relaxation time, possibly indicating that acute sleep deprivation affected primarily early left ventricular diastole, i.e., the energy-consuming process of diastole, which for example is also affected by myocardial ischemia [13] or changes in afterload [14]. The only finding which does not fit into the picture is the lack of change (not even a trend) in the early diastolic mitral annular velocity $(e)$, which is currently regarded as one of the best if not the best non-invasive measures of left ventricular relaxation [15]. However, given that regional differences in $e^{\prime}$ along the mitral annulus are well known, i.e., the lateral $e^{\prime}$ is typically higher than the septal $e^{\prime}$ [15], the neutral findings regarding $e^{\prime}$ in the present study may be explained by the fact that $e^{\prime}$ was measured at the septal annulus only, whereas a global left atrial strain rate was calculated from measurements obtained at four different left atrial sites. Previous studies have shown that acute sleep deprivation can induce endothelial dysfunction [4]. Unfortunately, no measures of vascular function have been obtained in the present study. It is possible but remains hypothetical that by an increase in left ventricular afterload and subsequent impairment in left ventricular relaxation, acute sleep deprivation caused a reduction in the left atrial early diastolic strain rate. These changes were subtle, and there was not even evidence of a compensatory increase in left atrial contraction (left atrial booster function) and no change in left atrial systolic strain rate. In other words, acute sleep deprivation seems to induce a transient very mild form of impaired left ventricular relaxation with subsequent impairment of the left atrial conduit function. Importantly, similar chronic left atrial strain patterns have been observed in normal aging [11] and mild hypertension [12]. Thus, the findings of the present report are sound. Still, further studies will have to expand these findings by exploring the underlying mechanisms.

Finally, what are the clinical implications? The question is whether or not acute sleep deprivation studies can serve as models for what is happening during chronic sleep deprivation. The data are relatively clear for apneic sleep deprivation, i.e., OSA: the more severe the OSA is, the more relevant are the cardiovascular consequences even in terms of hard clinical endpoints [16]. The situation is not so clear with regard to chronic sleep deprivation or, more correctly spoken, chronically short sleeping times. There is indeed an association between short sleeping time (less than $6 \mathrm{~h}$ ) and an increased risk of hypertension [2]. However, the relationship between sleeping time and the risk of hypertension is in fact $U$ shaped [2]. To make the story more complicated, those who have short sleeping times but do not feel that it is too short do not have an increased cardiovascular risk [17]. Thus, it is very likely that the acute cardiovascular response to sleep deprivation is modulated in many ways if short sleeping time becomes more chronic to protect otherwise healthy people from adverse long-term consequences. Another open question, however, is whether or not the observed phenomenon could play a role in patients with preexisting cardiac disease, e.g., paroxysmal atrial fibrillation or heart failure, in that acute sleep deprivation could trigger an episode of atrial fibrillation or ventricular arrhythmia, respectively, as the heart in these patients may be more vulnerable than in healthy people.

In summary, the findings by Acar et al. are novel and exciting and have opened the door for further research in another aspect of the conundrum of sleep and cardiovascular disorders.

Conflict of interest None.

\section{References}

1. Kasai T, Bradley TD (2011) Obstructive sleep apnea and heart failure. Pathophysiologic and therapeutic implications. J Am Coll Cardiol 57:119-127

2. Gottlieb DJ, Redline S, Nieto FJ, Baldwin CM, Newman AB, Resnick HE, Punjabi NM (2006) Association of usual sleep duration with hypertension: the Sleep Heart Health Study. Sleep 29:1009-1014

3. Yaggi HK, Araujo AB, McKinlay JB (2006) Sleep duration as a risk factor for the development of type 2 diabetes. Diabetes Care 29:657-661

4. Sauvet F, Leftheriotis G, Gomez-Merino D, Langrume C, Drogou C, Van Beers P, Bourrilhon C, Florence G, Chennaoui M (2010) Effect of acute sleep deprivation on vascular function in healthy subjects. J Appl Physiol 108:68-75

5. Dettoni JL, Consolim-Colombo FM, Drager LF, Rubira MC, Souza SB, Irigoyen MC, Mostarda C, Borile S, Krieger EM, Moreno H Jr, Lorenzi-Filho G (2012) Cardiovascular effects of 
partial sleep deprivation in healthy volunteers. J Appl Physiol 113:232-236

6. Zhong X, Hilton HJ, Gates GJ, Jelic S, Stern Y, Bartels MN, Demeersman RE, Basner RC (2005) Increased sympathetic and decreased parasympathetic cardiovascular modulation in normal humans with acute sleep deprivation. J Appl Physiol 98:2024-2032

7. Esen O, Akcakoyun M, Acar G, Bulut M, Alizade E, Kargin R, Emiroglu MY, Yazicioglu MV, Gemici K, Esen AM (2011) Acute sleep deprivation is associated with increased atrial electromechanical delay in healthy young adults. Pacing Clin Electrophysiol 34:1645-1651

8. Sari I, Davutoglu V, Ozbala B, Ozer O, Baltaci Y, Yavuz S, Aksoy M (2008) Acute sleep deprivation is associated with increased electrocardiographic P-wave dispersion in healthy young men and women. Pacing Clin Electrophysiol 31:438-442

9. Ozer O, Ozbala B, Sari I, Davutoglu V, Maden E, Baltaci Y, Yavuz S, Aksoy M (2008) Acute sleep deprivation is associated with increased QT dispersion in healthy young adults. Pacing Clin Electrophysiol 31:979-984

10. Acar GO, Akcakoyun M, Sari I, Bulut M, Alizade E, Ozkan B, Yaziciogluu MV, Alici G, Avci A, Kargin R, Esen AM (2012) Acute sleep deprivation in healthy adults is associated with a reduction in left atrial early diastolic strain rate. Sleep Breathing (in press)

11. Boyd AC, Richards DA, Marwick T, Thomas L (2011) Atrial strain rate is a sensitive measure of alterations in atrial phasic function in healthy ageing. Heart 97:1513-1519
12. Eshoo S, Boyd AC, Ross DL, Marwick TH, Thomas L (2009) Strain rate evaluation of phasic atrial function in hypertension. Heart 95:1184-1191

13. Carluccio E, Biagioli P, Alunni G, Murrone A, Leonelli V, Pantano P, Vincenti G, Giombolini C, Ragni T, Reboldi G, Gentile F, Ambrosio G (2009) Effect of revascularizing viable myocardium on left ventricular diastolic function in patients with ischaemic cardiomyopathy. Eur Heart J 30:1501-1509

14. Borlaug BA, Melenovsky V, Redfield MM, Kessler K, Chang HJ, Abraham TP, Kass DA (2007) Impact of arterial load and loading sequence on left ventricular tissue velocities in humans. J Am Coll Cardiol 50:1570-1577

15. Nagueh SF, Appleton CP, Gillebert TC, Marino PN, Oh JK, Smiseth OA, Waggoner AD, Flachskampf FA, Pellikka PA, Evangelisa A (2009) Recommendations for the evaluation of left ventricular diastolic function by echocardiography. Eur J Echocardiogr 10:165-193

16. Marin JM, Corrizo SJ, Vicente E, Agusti AG (2005) Long-term cardiovascular outcomes in men with obstructive sleep apnoeahypopnoea with or without treatment with continuous positive airway pressure. Lancet 365:1046-1053

17. Chandola T, Ferrie JE, Perski A, Akbaraly T, Marmot MG (2010) The effect of short sleep duration on coronary heart disease risk is greatest among those with sleep disturbance: a prospective study from the Whitehall II cohort. Sleep 33:739-744 